\title{
WebGIS Enabled Facility Mapping and Identification - A Cost Effective Solution
}

\author{
Rabi Sankar Mondal \\ Dept. of Computer Science \\ \& Engineering, GCECT, \\ Kolkata, India
}

\author{
Debasish Chakraborty \\ RRSC-East, NRSC, ISRO, \\ New Town, Kolkata, India
}

\author{
Arati Paul \\ RRSC-East, NRSC, ISRO, \\ New Town, Kolkata, India
}

\author{
Kibria Dastagir Dafadar \\ Department of Computer Application \\ Narula Institute of Technology, Agarpara, \\ Kolkata, India
}

\begin{abstract}
WebGIS publicizes and shares geospatial data through Internet for visualizing and analyzing the area of interest. In order to make a cost effective implementation, operation and maintenance of geospatial data over the Web for facility mapping and detection, a cheaper yet feature rich alternative to commercial software is required which can be fulfilled by existing free and open source software (FOSS) for geographic information (FOSSGIS) systems and FOSS for web development respectively. In an effort to understand the FOSS features and capabilities, reviewed many of the state-ofthe-art FOSS software projects in the context of those that have been used for sharing geospatial data. Subsequently, proposed an approach for visualizing facilities in Web environment using open source GIS software to display and identify locations of establishments. The application enables to visualize the spatial distribution of specific type of establishment in state/ district. The navigation tools (Zoom-in, zoom-out, and pan) and measurement tools are included in the application for navigating the map and measuring distance/area in the map respectively.
\end{abstract}

\section{Keywords}

WebGIS, FOSSGIS, FOSS, map, facility, Internet

\section{INTRODUCTION}

WebGIS is a type of GIS (Geographic Information System) that combines a geographical information system and web technology. The reach of the GIS system multiples manifold when it is implemented through the internet. Not only does it get a greater user set, it also means that the user need not invest in a resource intensive machine to access the WebGIS system. This makes WebGIS both cheap and more readily accessible. There are number of Free Open Source and OGC (Open Geospatial Consortium) complain GIS softwares are presently being used to minimize the costs encountered in developing WebGIS application. Free and open-source software (FOSS) is computer software that can be classified as both free software and open-source software. The benefits of using FOSS can include decreasing software costs, increasing security and stability (especially in regard to malware), protecting privacy, and giving users more control over their own hardware [5]. Open Geospatial Consortium [13] is an international, voluntary consensus standards organization that is leading the development of standards for geospatial and location based services. Web Feature Service (WFS), Web Map Service (WMS) and Web Coverage Service (WCS) are the three important OGC standards. OGC-WMS is concerned with serving raster and vector data as maps (images) in PNG, GIF or JPEG format, whereas OGC-WFS allows direct access to the vector data including reading, writing, and updating. OGC-WCS is used to serve or share raster or image layers on the web [8].

In an endeavour to realize the web of FOSS features and capabilities, we reviewed the FOSS software projects in the context of those that have been used to develop WebGIS apps that have been published in the literature in the last few years. Subsequently, proposed suitable FOSS for developing application for mapping and identifying the facilities of an organisation, viewing satellite image and non-spatial data efficiently in web environment.

There is an ample of FOSSGIS available that can be used to acquire, modify, store, visualize, and analyze spatial data in web environment. We organized the review into three categories: Geospatial data server for sharing spatial data, mapping libraries for visualizing spatial datasets, spatial analysis for geoprocessing, database for storing non-spatial data and Web development Programming language.

\subsection{Geospatial Data Servers}

Geospatial data server is software that publishes the spatial data. It makes spatial data available in web-friendly formats. This is done by offering the data or visualizations of the data as OGC standardized web services, which can then be rendered on a web page in a browser using a mapping library or plug-in (discussed in the next section). The primary OGC standards that are applicable to geospatial data servers are the web mapping service (OGC-WMS), web feature service (OGC-WFS), and web coverage service (OGC-WCS). We found three Geospatial data server software namely GeoServer, UMN MapServer, and MapGuide were used in the web apps projects and reported in $[2,3]$.

GeoServer is an open source Mapping Server written in Java that allows users to share and edit geospatial data and display it to the entire world [6]. It publishes data from any major spatial data source using open standards. GeoServer enables to connect to a data source such as PostGIS, ArcSDE, Oracle, and DB2 [6]. GeoServer is built with the help of Geo tools, which is an open source Java library that provides GIS support for spatial data types such as vector and raster layer [6]. It implements in it the Web Map Service (WMS) standard, which helps it in creating maps in a variety of output formats. GeoServer include integrated Open-Layers and Google Earth support, GeoWebCache automated spatial caching, tile mapping. In addition, the use of OpenLayers, which is a free 
mapping library, in GeoServer makes map generation very quick and easy.

The UMN MapServer (University of Minnesota MapsSrver) commonly called just "MapServer" is an internet map server, a server-side piece of software which renders GIS data sources into cartographic map products on-the-fly [11]. MapServer supports numerous raster and vector data formats via the inbuilt libraries including TIFF, GeoTIFF, ESRI shapefiles, and PostGIS. MapServer runs on all major operating systems and work with almost any web server. Supports popular Open Geospatial Consortium (OGC) standards including WMS, WFS and WCS.

MapGuide enables users to develop and deploy web mapping applications and geospatial web services [10]. MapGuide features an interactive viewer that includes support for feature selection, property inspection, map tips, and operations. MapGuide includes an XML database for managing content, and supports most popular geospatial file formats, databases, and standards.

\subsection{Mapping Libraries}

Mapping libraries are needed to visualize spatial data in a web environment. Mapping libraries or plug-in consume data from OGC-WMS, OGC-WFS, and OGC-WCS web services and render the maps for presentation in a client (i.e.: web browser). The mapping libraries used in the web apps reviewed for this paper are all JavaScript libraries that run in web browsers. We found two mapping libraries reported in $[2,20]$ : OpenLayers and Google Maps ${ }^{\mathrm{TM}}$.

OpenLayers is a web-mapping client library for rendering interactive maps on a web page [7]. It is a pure Java-Script library for building rich web-based geospatial applications similar to Google Maps. OpenLayers is capable of rendering vector and raster data from a variety of formats including GeoJSON, OGC-KML, OGC-GML, and OGC web services. OpenLayers provides methods for drawing on the map and editing data interactively. It allows developers to use a variety of services for base maps including Open Street Map, Bing, MapQuest, and Google. It does not require a plug-in.

Google Maps provides a library that allows users to draw shapes on the map and edit spatial data interactively. Google Maps is capable of displaying spatial data in OGC-KML format. Alternatively, data can be added dynamically using the JavaScript API. It does not require a browser plug-in.

\subsection{Spatial Analysis}

Spatial analysis in WebGIS applications can be achieved by using software projects that implement the OGC-WPS (OGCWeb Processing Service) standard. The WPS offers a simple web-based method of finding, accessing, and using all kinds of calculations and models. The OGC-WPS interface standard provides rules for standardizing how inputs and outputs (request and responses) for invoking geospatial processing services, such as polygon overlay, as a Web service [14]. In this section we present the FOSSGIS software that was used to support spatial analysis in the web apps reported in the literature.

GeoServer provides a full implementation of OGC-WPS in addition to the spatial data publishing services. The processes can be called with GeoServer resources as inputs and they can output to new GeoServer resources.

The 52_ North WPS project represents a full implementation of the OGC-WPS standard [21]. 52_North WPS provides an extensible, pluggable framework for publishing geoprocessing algorithms as web services.

PyWPS is an implementation of OGC-WPS written in Python. Like any OGC-WPS, PyWPS does not process data itself, rather, it provides the link between the web and the local tools on the server such as GRASS, GDAL, and R scripts [4].

\subsection{Database}

Database is to store non-spatial data in a file system that is suitable for large datasets with several features and provide and efficient mechanism to store, query, analyze and update these data. We reviewed the open source database namely PostgreSQL, MySQL and SQL Lite respectively that are reported in literature [19].

PostgreSQL is a powerful, open source database [15]. It runs on all major operating systems, including Linux, UNIX, and Windows. It is reliable, stable, cross platform support, extensible and can handle spatial data. PostGIS is an open source software program that adds support for geographic objects to the PostgreSQL [16].

MySQL is a database management system used and runs on a server [9]. It is ideal for both small and large applications. It is very fast, reliable, and easy to use. It enables to handle data type like Numeric, String, Date and time and store 64 terabytes data [9].

SQL Lite is a library that implements a selfcontained, serverless, zero-configuration, transactional SQL database engine. The code for SQL Lite is in the public domain and is thus free for use for any purpose, commercial or private [17].

\subsection{Web Development Programming Language}

The programming languages are used in web development to make websites more dynamic and to handle advanced logic beyond simply returning static HTML. We reviewed the programming languages namely PHP and Java respectively that are frequently used.

PHP (Hypertext Preprocessor) is a very popular scripting language that is especially suited for web development. PHP is embedded in the HTML pages of the website and the code is executed on the server when the web page is requested. It includes support for a wide range of databases via ODBC. It also has many extensions that add common web functionality such as managing sessions and cookies, user authentication, and file uploads [18].

Java technologies are free and open source and provide a powerful, cross-platform development environment for creating and running large-scale, multi-tiered, scalable, reliable, and secure network applications [12].

\section{APPLICATION ARCHITECTURE}

Three tier architecture is followed for implementing the application. The first and second tires include client (Browser) and application server respectively, whereas Geospatial data server (GeoServer) and database server (PostgreSQL/ PostGIS) reside in third tier (figure 1). The application server uses Apache web server [1] to access the client requests through Hyper Text Transfer Protocol (HTTP). Subsequently the application server analyses the request and sends the query either/both to GIS server or/and database server. On a successful query the database and/or GIS server replies to the application server. Finally the application server presents the 
result to the client in a customized form through HTTP. Apache is proposed as the web server because it is the most popular PHP developer package and independent to operating system. It is also easy to install and configure and completely free of charge [1]. GeoServer software is used for sharing the map since the integrated mapping library "OpenLayers" in GeoServer makes map generation very quick and provides a facility of spatial analysis and does not require a plugin [6]. PostgreSQL is used as the database because its concurrency control is better than SQLite and MySQL since it employs MVCC (Multi-version Concurrency Control) and it is designed for high volume environments. It runs on all major operating systems, including Linux, UNIX and Windows. Moreover it can contain both spatial and non-spatial data. It never crashes even under high activity operations and it adds support for geographic objects allowing location queries to be run in SQL [15]. The PHP is proposed for developing the interface of the application to view, customized retrieval, and query on geospatial data for specific needs. Since PHP is especially suited for web development and support a wide range of databases via ODBC.

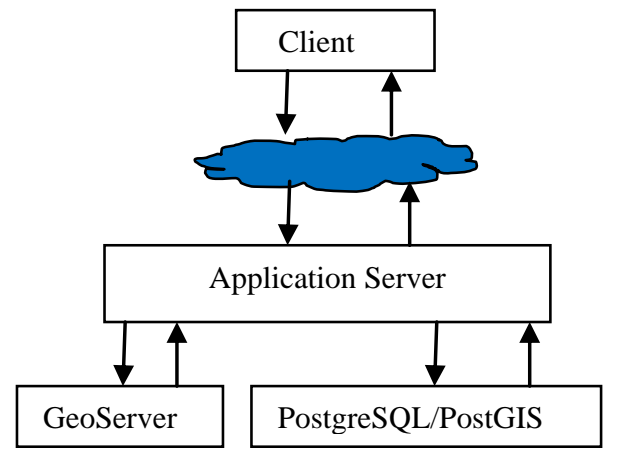

Figure 1: System Architecture of application

\section{METHODOLOGY}

The locations of office establishments are converted into spatial layers. Subsequently type and address of the offices are integrated with spatial layers and stored in PostgreSQL/PostGIS database $[15,16]$. These data are shared over the Internet in OGC-WMS standard using GeoServer [6]. Consequently the Web enabled office establishment visualization facility is developed using Open Layer, HTML, PHP and Java scripts. The PHP accesses and shares the data stored in PostgreSQL/PostGIS and Java scripts are used to consume and present the services in a customized form to the user.

\section{APPLICATION INTERFACE}

An interactive user friendly interface is developed for visualizing the locations of office establishments over the web. Five types of office establishments such as type-1 (i.e. Regional office) / type -2 (i.e. District Development office) / type -3 (i.e. Project management Unit) / type -4 (Training Establishments office) / type -5 (Sub office) available in a state / district are used in this study. The number of various types of establishment mapped in this study is shown in figure 2.

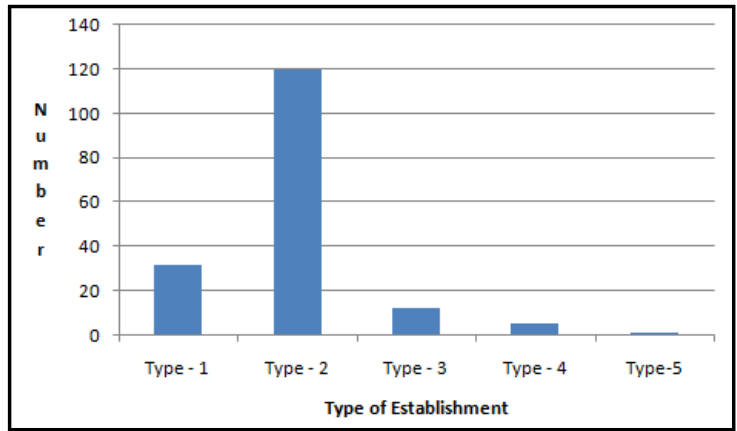

Figure 2: The number of various types of office establishments locations mapped in this study

The major components of the interface are categorized into viewer, query and tools. The viewer enables to display and overlay the satellite image and maps. It facilitates to display the office establishment locations along with its address. Provision is kept for displaying geographic distribution of various types of office establishments throughout the region of interest along with legend. The tools viz. zoom in, zoom out, zoom to full extent, selective zoom, re-centre tool and pan are provided for navigation. The measurement tools are developed for measuring the distance and area in the map. The print map tool is included to print the map. The display tool made available in the interface to switch the current display of the viewer to map/ satellite image/ hybrid (map overlaid on image) mode. The "query" enables user to visualize the specific type of establishments such as type-1) / type -2 / type -3 / type $-4 /$ type -5 available in a state / district. Figure 3 is showing the output of the query carried out for displaying geographic distribution of Type -3 establishments available in the database.

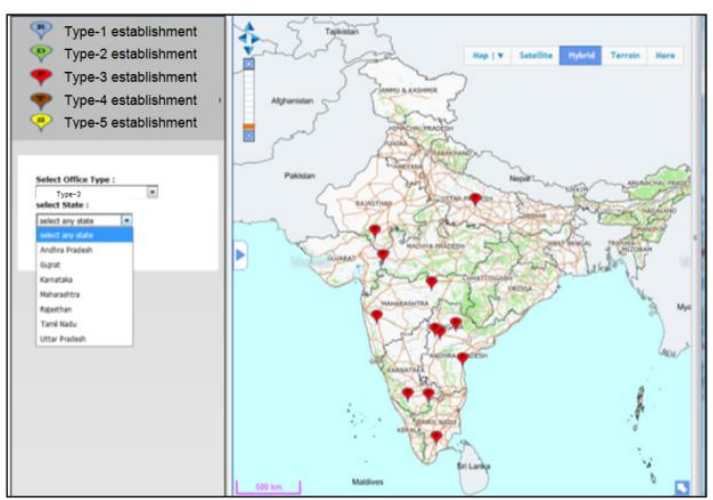

Figure 3: Showing geographic distribution of Type -3 establishments

\section{CONCLUSION}

We performed a review of WebGIS application projects that were published in the peer-reviewed literature in last few years to find out which FOSSGIS and FOSS Web development software was used to develop the web application. The software review includes FOSSGIS geospatial data server, mapping libraries and spatial analysis. This review enabled us to understand the potentiality of various FOSSGIS, FOSS Web development software and database. The proposed approach for sharing and mapping services using PostgreSQL, Geoserver, Apache and PHP enabled facility mapping and identification. The future work will extend in developing national level WebGIS enabled facility mapping and gap analysis using the proposed method. 


\section{ACKNOWLEDGEMENT}

Authors are thankful to the Director, NRSC, Hyderabad, India, CGM, RCs, NRSC, Hyderabad, India and GM, RRSCEast, NRSC, Kolkata for their guidance and support on carrying out this research.

\section{REFERENCES}

[1] Appache, 2015, Appache Documentation. From. http://www.apachefriends.org/.

[2] Blodgett, D., Booth, N., Kunicki, T., Walker, J., Lucido, J. ( 2012) Description of the US Geological Survey Geo Data Portal Data Integration Framework. Selected Topics in Applied Earth Observations and Remote Sensing IEEE J. 5 (6): 1687-1691.

[3] Cau, P., Manca, S., Soru, C., Muroni, D., Gorgan, D., Bacu, V., Giuliani, G. (2013) An interoperable, GISoriented, information and support system for water resources management. Int. J. Adv. Comput. Sci. Appl. 3 (3): 75-82. http:// dx.doi.org/10.14569/SpecialIssue.2013.030309.

[4] Castronova, A.M., Goodall, J.L., Elag, M.M., 2013. Models as web services using the Open Geospatial Consortium (OGC) Web Processing Service (WPS) standard. Environ. Model. Softw. 41, 72-83. http://dx.doi.org/10.1016/J.Envsoft.2012.11.010.

[5] Free software foundation, 2015,http://www. opensource.com/resources/what-open-source.

[6] GeoServer, 2015. GeoServer Documentation. From. http://docs.geoserver.org/.

[7] Hazzard, E. (2011) OpenLayers 2.10: Beginner's Guide. Packt Publishing, Birmingham.

[8] Jeff de la Beaujardiere. (2002) Web Map Server Implementation Specification, OGC 01-068r3 version 1.

[9] MySQL, 2015, MySQL Documentation. From. http://dev.mysql.com/doc/refman/4.1/en/what-ismysql.html.
[10] Mapguide, 2015, Mapguide Documentation. From. http://mapguide.osgeo.org/.

[11] MapServer, 2015, MapServer Documentation. From. http://www.osgeo.org/mapserver.

[12] Oracle, 2012. Your First Cup. Retrieved 28 October 2014, from. http://docs.oracle.com/javaee/6/firstcup/doc/gkhoy.html.

[13] OGC, 2015, OGC Documentation. From. http://www.opengeospatial.org/ogc

[14] OGC-WPS, 2015, OGC-WPS documentation From http://www.opengeospatial.org/standards/wps.

[15] PostgreSQL, 2015, PostgreSQL Documentation. From. http://www.postgresql.com.

[16] PostGIS, 2015, PostGIS Documentation. From. http://postgis.net/.

[17] SQL Lite, 2015, SQL Lite Documentation. From. http://www.sqlite.org/about.html.

[18] Royappa, A.V. (2000) The PHP web application server. J. Comput. Sci. Coll, 15 (3), 201-211.

[19] Sarup J. \& Shukla V. (2012) Web-Based solution for Mapping Application using Open-Source Software Server, International Journal of Informatics and Communication Technology (IJ-ICT), 11 (2):. 91-99.

[20] Wan, Z., Hong, Y., Khan, S., Gourley, J., Flamig, Z., Kirschbaum, D., Tang, G. (2014)A cloud-based global flood disaster community cyber-infrastructure: development and demonstration. Environ. Model. Softw. 58: 86-94.

[21] 52 _North, 2014. Home e 52_North Initiative for Geospatial Open Source Software GmbH. Retrieved 12 March 2013, from. http://52north.org/. 